\title{
On a small Nebula close to M. 57.
}

By E. E. Barnard.

In examining the annular Nebula of Lyra (M. 57) with the 36 inch on October $2^{\text {nd }}$, I noticed a rather conspicuous nebula in the field with it, and north preceding.

As I have seen no record of this object, I bave measured its position referred to the centre of the Ring Nebula, or rather with reference to the small star in the middle of the ring.

October $2^{\text {nd }}$ the $\Delta \alpha$ and $\Delta \delta$ were measured and October 8 the position angle and distance.

$$
\begin{array}{rcc} 
& \multicolumn{2}{c}{\text { Neb. }- \text { M. } 57} \\
1893 & \Delta \alpha & \Delta \delta \\
\text { Oct. } 2 & -206.5+131.1 \\
8 & -202.9+133.3 \\
\cline { 2 - 3 } & -204.7+132.2
\end{array}
$$

The $\Delta \alpha$ and $\Delta d$ for October 8 , being derived from the following position angle and distance:

$$
\text { P. A. }=3 \circ 3^{\circ} \cdot 3 \quad \text { Dist. }=242.8
$$

This nebula is about $1 \mathrm{~s}^{\circ}$ diameter. Not round. A little brighter in the middle. About $14^{\text {th }}$ magnitude.

Inclosed is a sketch of its position (diam. of field $6^{\circ} \pm$ ).

I have not shown all the stars that are easily seen in this field.

Mt. Hamilton 1893 Oct. 8.

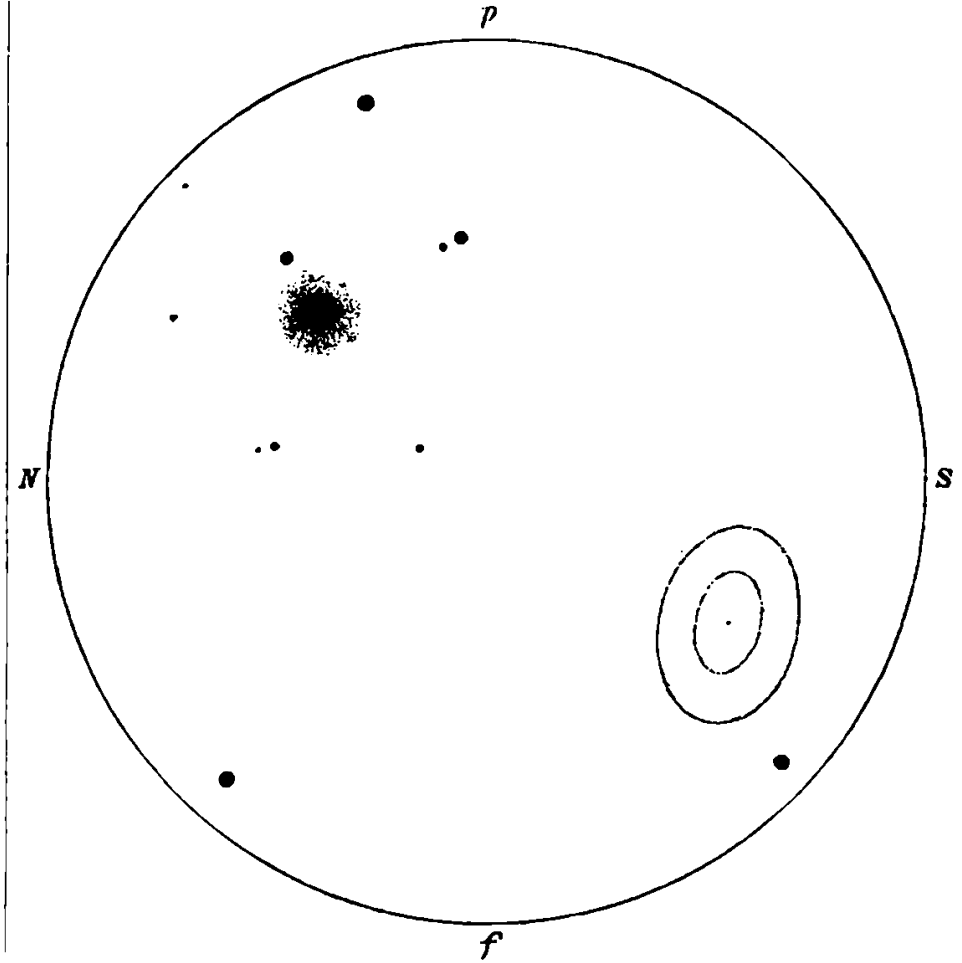

E. E. Barnard.

\section{A Special Case of an Error, in the observed Time of a Star's Transit,} which is a Function of the Star's Magnitude.

\section{By F. M. Schaeberle.}

In January $189 \mathrm{I} I$ received a letter from Professor Auwers of Berlin in which he informed me that from a discussion of a series of observations sent to him it appeared that $I$ observed bright stars in transit earlier than faint ones, and that the difference in time amounted to about 0.02 for every whole magnitude.

Several series of observations made for the special purpose of investigating this particular subject plainly demonstrated the general correctness of Dr. Auwers' conclusions.

In this special investigation it was necessary to eliminate as far as practicable errors due to a change of conditions during the observations on different stars; the work, therefore, was so planed that each star observed during its transit furnished all the data for obtaining an independent value of the desired quantity.

To reduce the brightness of a star of known magnitude, wire screens were made use of. A single thickness or sheet of the fine wire screen (manufactured by the Repsold's) when placed directly in front of the meridian circle objective was found to reduce the brightness of the stars about two magnitudes. Two and three of these screens superposed reduced the brightness by about four and six magnitudes respectively.

The program adopted was as follows.

Each star (B. J.) was observed over six wires symmetrically situated with reference to the middle wire. If the transits over the furst three wires were observed with the screen, then the remaining three transits were observed without the screen, and vice versa.

As only naked eye stars of known magnitude were to be used and as stars down to the tenth magnitude can be readily observed only two compound screens were required to obtain the desired range in magnitude. One compound screen was made of two sheets and the other of three sheets superposed so that the brightness could be reduced about four and six magnitudes respectively.

During the star's transit an Assistant, Professor Treat (at the time a special student here) with the screens in readiness, would at a given signal cover or uncover the objective, thus leaving the observer undisturbed at the telescope.

If the star was brighter than the third magnitude the compound screen containing three sheets was to be used; 